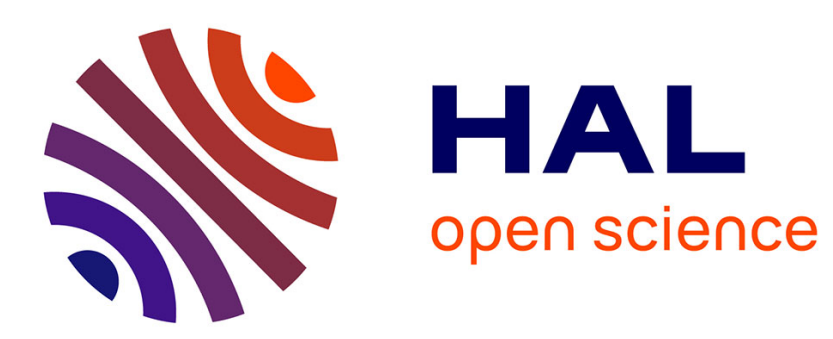

\title{
Démystifier le mystique: Joséphin Péladan et les cercles rosicruciens à l'épreuve du rire fin de siècle
}

\author{
Damien Delille
}

\section{To cite this version:}

Damien Delille. Démystifier le mystique: Joséphin Péladan et les cercles rosicruciens à l'épreuve du rire fin de siècle. Romantisme: la revue du dix-neuvième siècle, 2012, 156, pp.87-100. halshs-01796700

\section{HAL Id: halshs-01796700 \\ https://shs.hal.science/halshs-01796700}

Submitted on 21 May 2018

HAL is a multi-disciplinary open access archive for the deposit and dissemination of scientific research documents, whether they are published or not. The documents may come from teaching and research institutions in France or abroad, or from public or private research centers.
L'archive ouverte pluridisciplinaire HAL, est destinée au dépôt et à la diffusion de documents scientifiques de niveau recherche, publiés ou non, émanant des établissements d'enseignement et de recherche français ou étrangers, des laboratoires publics ou privés. 


\section{Démystifier le mystique : Joséphin Péladan et les cercles rosicruciens à l'épreuve du rire fin de siècle}

Le renouveau spiritualiste de la fin du XIX ${ }^{\mathrm{e}}$ siècle, en marge d'une Troisième République rompant avec son Église, se traduit par la multiplication de ce que l'occultiste Jules Bois nomme, en 1894, les " petites religions ${ }^{1}$ ". Son enquête consacrée aux milieux ésotériques, bouddhistes, satanistes et mystiques chrétiens fait la lumière sur les superstitions et autres canulars spirites qui émaillent l'époque, alors qu'un puissant mouvement anticlérical s'ingénie à dénoncer ces expériences mystiques que l'Église n'arrive plus à maîtriser. L'entreprise de démystification attaque ainsi le mystificateur, défend le mystifié, soulignant au passage combien ces impostures cachent souvent d'érotiques desseins en immédiate contradiction avec l'idéalisme ambiant.

Deux visions sociétales de l'identité masculine s'opposent (idéalisme versus populisme) avec, comme vecteur commun, un antiféminisme exacerbé. Les mystifications de Joséphin Péladan, déjouées par ceux-là mêmes qui tendent à le ridiculiser, permettent d'abord d'apprécier la réception des cercles artistiques de la Rose-Croix. Mais les caricatures des revues satiriques mêlées aux discours sur la dégénérescence conduisent in fine à disqualifier le projet symboliste lui-même. Cette histoire comparée renouvelle ainsi les études sur la masculinité, en tirant profit de l'histoire visuelle des sciences et de ses répercussions dans l'imaginaire populaire. C'est aussi l'objet de ces démystifications iconographiques, qui montrent le glissement progressif de la peur de l'efféminement à une stigmatisation de l'homosexualité. Tel semble être le prix à payer par des pratiques trop mystiques pour être honnêtes.

\section{DÉMYSTIFIER L’ÉROTOMANIE IDÉALISTE}

La volonté de Jules Bois de "désocculter l'occultisme " "s'inscrit dans le cadre du renouveau de l'ésotérisme chrétien, au moment où les règles de l'Église romaine sont disputées, sur fond de séparation de l'Église et de l'État. L'efflorescence de ces petites Contre-Églises est la proie de l'anticléricalisme laïc, illustré notamment par les très populaires mystifications de Léo Taxil. En révélant les supercheries de

1. Jules BoIs, Les petites religions de Paris, Paris, L. Chaillet, 1894.

2. J. Bouvéry, Le Moniteur Spirite et Magnétique, n 6, juin 1891, p. 125-127. 
l'interprétation chrétienne de l'évolution, à travers les impostures de la fausse Diana Vaughan, Taxil fait passer les occultistes pour des agents du diable ${ }^{3}$. Ces « pseudoreligions ${ }^{4}$ ", parodies de la religion officielle, deviennent selon Jules Bois l'épicentre d'un nouveau féminisme capable de régénérer l'humanité. Influencé par la Société théosophique, créée en 1875 par Héléna Blavatsky et sa branche anglaise, dirigée par Lady Caithness, Bois restaure une image acceptable de ces groupuscules officiant à Paris.

Joséphin Péladan prend une part active à ces mouvements, en rejoignant dès 1887 une branche ancienne de la Rose-Croix catholique, associée au mouvement Kabbalistique de Stanislas de Guaita. Se séparant de cette influence maçonnique en 1890, il fonde l'Association de l'Ordre du Temple de la Rose-Croix ${ }^{5}$, prémisse de la première "Geste esthétique » du Salon de la Rose-Croix, en mars 1892. Le Charivari, prompt à dénoncer par l'image les nouvelles supercheries des milieux spirites, se fait l'écho de ce renouveau de l'art sacré, en imaginant dans son numéro du dix septembre les mises en scène sacramentelles de cette nouvelle religion de l'Art. Aux priants devant les tableaux représentant des croix, se mêle l'interprétation anticléricale du spiritualisme religieux, faite de "vague à l'âme " et d' " harmonie céleste ${ }^{6}$ ".

Tout le paradoxe de l'idéalisme symboliste de Péladan réside dans sa critique de la décadence moderne (matérialisme, décadence de la culture latine, émancipation féminine), conjuguée à une fascination non dissimulée pour cette décadence (spiritualité exaltée, troubles sexuels exacerbés). C’est cette ambiguïté qui va être dénoncée avec violence par ses adversaires. Péladan subit des attaques répétées contre son image publique de mage, les extravagances douteuses de ses apparitions et le caractère apologétique de ses interventions. Affublé du titre de $\mathrm{Sâr}^{7}$ et du prénom néo-babylonien de "Mérodack" (de Baladan Mérodack, roi de Babylone), il se présente "drapé d'un burnous noir en poil de chameau filamenté de fils d'or, en velours vieux bleu, botté de daim, et, comme Absalon, chevelu [...] la barbe ointe d'huile de cèdre ${ }^{8}$ ». Il combat ainsi la décadence sur le terrain de l'apparence, en lui opposant la figure supérieure du mage, devenu homme d'abstraction. Tout est une question d'attitude, dans le cadre d'une société industrielle où prédominent l'indistinction sociale et la fin des clivages aristocratiques : "dans un temps où les honneurs déshonorent, où la fonction signifie incapacité, il y a une sorte de vertu

3. Voir Jean-Pierre LAURANT, L'ésotérisme chrétien en France au XIX siècle, Lausanne, L'Âge d'homme, 1992.

4. René GuÉNON, Le théosophisme, histoire d'une pseudo-religion, Paris, Nouvelle librairie nationale, 1921.

5. Entre juin et août 1890, Péladan publie dans l'Initiation, deux articles instaurant le catholicisme de la Rose-Croix. Il fonde en août 1891, avec Léonce de Larmandie, Gary de Lacroze, Élémir Bourges et Antoine de la Rochefoucauld, l'association esthétique.

6. Le Charivari, 10 septembre 1891.

7. Selon l'entretien du Matin, 1er avril 1892. Péladan commente ainsi : « Au point de vue étymologique, Sar signifie sire, sieur, monsieur. Dans le sens divin du mot, cela veut dire tombé du ciel, ange déchu ; en d'autres termes : homme qui s'est fait lui-même ce qu'il est. » Cité dans Christophe BEAUFILS, Joséphin Péladan. Essai sur une maladie du lyrisme, Grenoble, Jérôme Millon, 1993, p. 214. p. 61.

8. Cité dans René-Louis Doyon, La Douloureuse aventure de Péladan, Paris, la Connaissance, 1946, 
publique à témoigner par un point du costume que l'on est soi, que l'on est libre, que l'on est hors cadre. Sauf sur le plan religieux qui est celui de l'abdication, le dandysme des décadences consiste à se différencier du nombre? ."

L'archétype de la "tête assyrienne ${ }^{10}$ ", imposée comme un double de l'auteur, nourrit le personnage de Mérodack, censé incarner la sagesse orientale et la distinction royale. Objet de caricature, ce profil assyrien reprend le topos orientaliste, au moment où les nouveaux sites de Darius et d'Artaxerxes à Suse sont découverts en $1884^{11}$. Le célèbre caricaturiste Caran d'Ache ${ }^{12}$ transforme ainsi Péladan l'assyrien en "image d'Épinal ", drapé d'une robe à l'antique et claironnant le renouveau religieux, à l'occasion des messes de Notre Dame ${ }^{13}$. L'illustration s'accompagne d'un article de Lucien Descaves sur "L'esprit nouveau ou le cléricalisme », qui dénonce les nouvelles entreprises religieuses. Se moquant ainsi de sa grandiloquence et sa religiosité feinte, Caran d'Ache souligne les rapports entre l'apparat religieux, le pouvoir politique (célébrer la messe de "M. Carnot ») et l'allure féminine de sa toge devenue robe de soirée. L'intéressé, ravi d'être mis au pinacle par ceux contre lesquels il se bat, se plaît dans ce rôle d'incompris caricaturé. Il commente à la troisième personne, dans la préface de son roman La Gynandre. "Le millier de calomnies et d'injures qu'il a collectionné, ce trimestre écoulé, l’ont blasé : il jouit aujourd'hui de ce rare sentiment : l'absolu de l'indifférence, en matière d'opinion ${ }^{14}$. "

L'excentricité vestimentaire, liée aux mouvances néo-médiévistes ${ }^{15}$, contribue à aiguiser la dénonciation carnavalesque. En associant les dates du carnaval au premier Salon, Péladan se retrouve parmi les costumes de 1892, affublé d'une fraise et d'une croix de l'ordre de Malte (en référence à l'ordre de la Rose-Croix), avec "Sar... Latan » pour épithète ${ }^{16}$. Ce jeu de mots dénonce la posture pseudo-religieuse d'un Péladan charlatan, débusqué à travers les ressorts imagés de sa longue plume derrière la main et par la position hautaine de son visage. Curieuse coïncidence, le tout premier Salon de la Rose-Croix se tient la même année que le premier bal des étudiants des Beaux-Arts, le fameux et fantasque bal des Quat'z'Arts, tous les deux associés à

9. Joséphin PÉladAn, L'Art idéaliste et mystique : doctrine de l'Ordre et du Salon des Rose-Croix, Paris, Chamuel, 1894, p. 58.

10. Joséphin PÉLADAn, Études passionnelles de décadence. Le Vice suprême, Paris, Librairie des auteurs modernes, 1884 , p. 83.

11. Voir Élisabeth Fontan (dir.), De Khorsabad à Paris. La découverte des Assyriens, Paris, Réunion des musées nationaux, 1994.

12. Sur la question des caricaturistes fin de siècle, voir Bertrand TILlIER, La républicature : la caricature politique en France, 1870-1914, Paris, CNRS Éd., 1997.

13. Le Journal, 19 mars 1894. On notera qu'une partie des nombreuses caricatures citées est tirée du fonds Péladan de la bibliothèque de l'Arsenal, Paris. Le fonds de Joseph-Aymé Péladan (de son vrai nom) a été versé à la bibliothèque en 1936, par la veuve du défunt, Christiana Péladan, née Taylor, et semble-t-il mis en forme par celui qui s'occupa de sa succession, René-Louis Doyon. Les différentes coupures de presse et caricatures, dont certaines rattachées au Courrier de la presse, démontrent que Péladan avait un abonnement pour recenser seul les occurrences le mentionnant, dans les journaux des années 1890. et XIII.

14. Joséphin PÉladAn, La Décadence latine. Éthopée. IX. La Gynandre, Paris, E. Dentu, 1891, p. XII

15. Voir Elisabeth EMERY et Laura MOROXITZ, Consuming the Past : the Medieval revival in fin-desiècle, Aldershot, Ashgate, 2003.

16. Le Charivari, 25 février 1892. 
une même déviances du trouble érotique. Dans l'une des caricatures du Salon, un bourgeois en visite est ébahi par la peinture d'une jeune fille au sein nu, respirant voluptueusement une fleur [fig. 1]. La légende indique que "sans M. Béranger [sic], nous devions avoir un nouveau bal des Quat'Sars ${ }^{17}$ ", rappelant la campagne menée par le sénateur René Bérenger, président de la Ligue de Défense de la Morale, qui avait poursuivi en justice l'année suivante le bal, pour suspicion d'orgies organisées ${ }^{18}$. Le jeu de mots s'accompagne ici d'une transcription visuelle tout aussi démonstrative, où le corps et l'environnement de la jeune fille sont figurés par des circonvolutions, proches stylistiquement du " serpentinisme » évoqué par une autre caricature d'Henri Avelot ${ }^{19}$. Dans cette charge, une jeune femme pleure devant les quatre versions de son portrait, peintes par un "moderniste », se plaignant que le serpentiste la représente " hystérique » et le symboliste, " toquée ». Le maniérisme rosicrucien est clairement attaqué sur le front de la psychopathologie de la création ${ }^{20}$.

L'opposition entre l'ordre moral républicain, conséquence de la défaite de 1870 , et les pratiques spiritualistes des années 1880 aiguise cette approche par la déviance des affects. Le savoir positiviste de la psychiatrie devient le fer de lance d'une politique anticléricale, qui relie les troubles de la personnalité à l'excès de religiosité ${ }^{21}$. L'un des représentants éminents de cette psychopathologie anticléricale, Jean-Martin Charcot, crée en 1885 la Société de Psychologie Physiologique, suivie de très près, avec l'aide de Paul Richet, par une Commission pour l'étude des phénomènes occultes, afin d'expliquer, par les seules lois expérimentales de la psychophysiologie, la folie et l'anormalité des phénomènes surnaturels. Les manifestations physiques de l'hystérie sont mises en étroite relation avec les phénomènes de croyance et d'hallucination, récusant tout effet magique de l'extase mystique et des états altérés de la spiritualité. Les manifestations mystiques des peintures religieuses vont ainsi trouver une explication pathologique ${ }^{22}$.

Voulant ancrer sa théorie de l'hystérie dans une nosographie, Charcot s'intéresse à l'équivalent masculin de l'hystérie et ouvre dès 1882, un "service des hommes " à la Salpetrière ${ }^{23}$. Contrairement à sa conception de l'hystérie féminine, liant érotomanie et symptôme hystérique, le rapport entre hystérie masculine et masturbation est rejeté par Charcot $^{24}$. Avant d'être une maladie, l'hystérie est un tempérament. Charcot impose

17. Fonds Péladan, MS 13412, feuillet 15, non identifié.

18. Voir Annie StORA-LAMARRe, L'enfer de la IIIe République. Censeurs et pornographes. 18811914, Paris, Imago, 1989.

19. Henri Avelot, « À travers les Quat'z'arts », 1896.

20. Sur ces questions, voir Rae Beth GoRDON, Ornament, Fantasy and Desire in Nineteenth Century French Literature, Princeton, Princeton University Press, 1992 et Debora L. Silverman, L'Art Nouveau en France. Politique, psychologie et style fin de siècle, Paris, Flammarion, 1994.

21. Voir sur ce point, Jan GoLDSTEIN, Consoler et classifier. L'essor de la psychiatrie française, Le Plessis-Robinson, Les empêcheurs de penser en rond, 1987 et Pascal LE MALÉFAN, Folie et spiritisme. Histoire du discours psychopathologique sur la pratique du spiritisme, ses abords et ses avatars (18501950), Paris, L'Harmattan, 1999.

22. Jean-Martin Charcot et Paul Richer, Les Démoniaques dans l'art, Paris, A. Delahaye et E. Lecrosnier, 1887.

23. On se reportera sur ce point à Mark S. MicAle, Hysterical Men. The Hidden History of Male Nervous Illness, Cambridge et Londres, Harvard University Press, 2008.

24. Voir Théodore TARCZYLO, Sexe et liberté au siècle des Lumières, Paris, Presses de la Renaissance, 1983. 


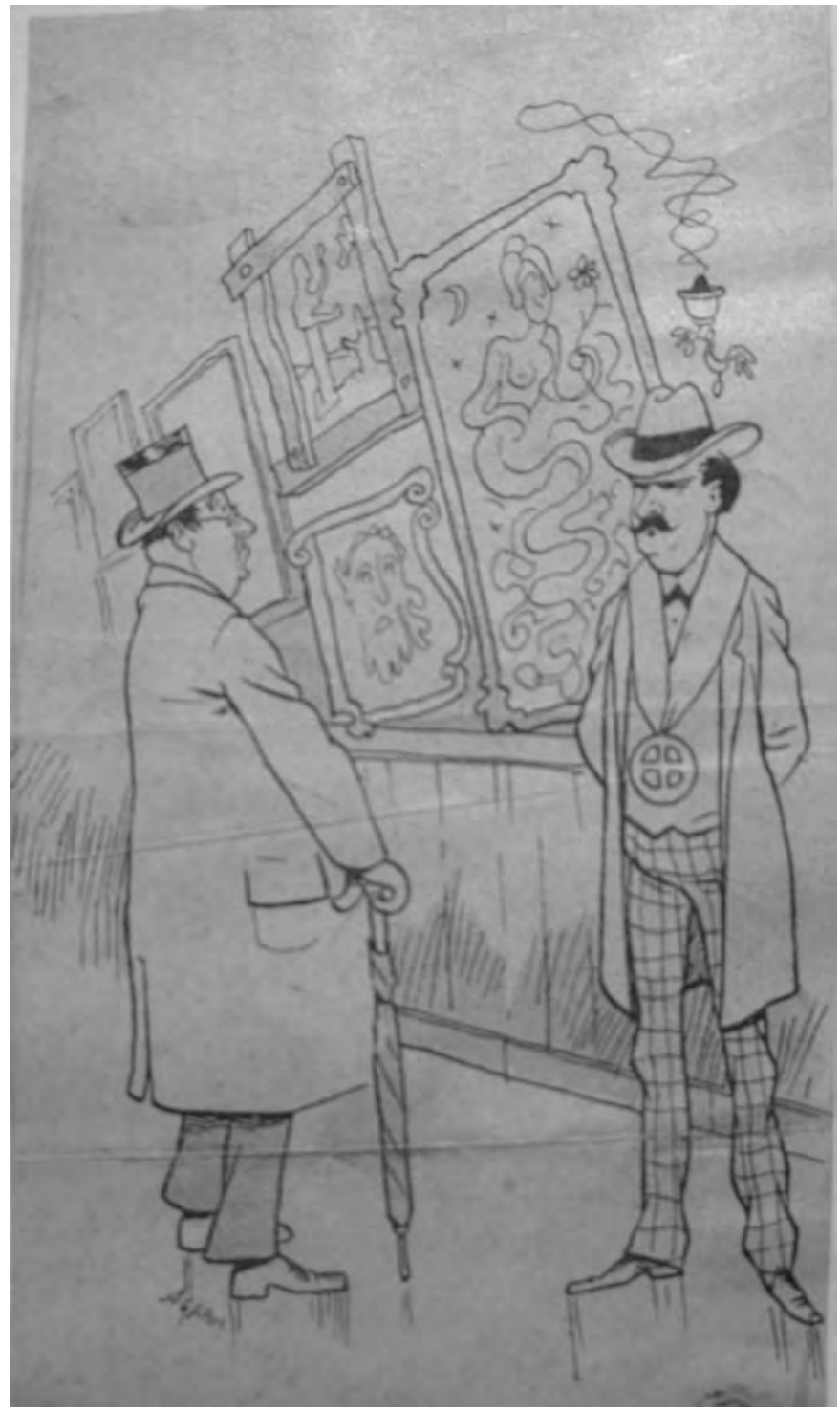

Figure 1. «Salon de la R + C », Fonds Péladan, MS 13412, feuillet.15, BNF.

(D.R.)

ainsi une distinction nette avec les cas d'adolescents, d'efféminés, d'homosexuels, de célibataires ou de prêtres, qui relèvent selon lui d'un dysfonctionnement physiologique extérieur à l'hystérie. L' " hystérie virile ${ }^{25}$ ", analysée dans une série d'études de cas,

25. Jean-Martin CHARCOT, Leçons sur l'hystérie virile, introduction par Michèle Ouerd, Paris, le Sycomore, 1984. 
va donc être découverte chez des cas d'ouvriers à l' « hétérosexualité » avérée, dont un contexte défini, comme les bruits d'un train ou une chute au travail, a provoqué l'hystérie. Les symptômes, contrairement aux expériences émotionnelles féminines (mort d'un proche, anxiété ou extase religieuse), vont être une hypermobilité ou le signe d'une mélancolie (attitude sombre et découragée).

En définissant l'hystérie virile à partir de la contraction musculaire et de l'apathie mélancolique, Charcot pose les bases d'une division genrée des attributs du masculin et du féminin ${ }^{26}$. Cependant, le trouble reste entier sur la distinction physiologique de l'inversion sexuelle, qui ne se classe dans aucune des deux divisions. Son étude avec Magnan d'un inverti sexuel, professeur d'université de trente et un ans, «bien charpenté " et qui se révèle "sexuellement très bien conformé ", souligne ses goûts féminins pour l'exaltation cultivée : la musique, la poésie, la toilette. Magnan avoue néanmoins que "regarder les parties génitales d'un homme beau et fort, telle a toujours été la volupté la plus grande pour moi $^{27}$ ". La peur du transfert sexuel et donc de la contagion du vice se retrouve au cœur de l'économie visuelle développée dans l'Iconographie de la Salpêtrière : la sur-représentation des clichés d'hystérie féminine compense le peu d'image d'hystérie masculine et la quasi-inexistence photographique des cas d'inversion sexuelle. Aussi concluent-ils qu' " on concède qu'un jeune homme efféminé puisse après des excès, des chagrins, des émotions profondes, présenter quelques phénomènes hystériformes ; mais qu'un artisan vigoureux, solide, non énervé par la culture [...] puisse devenir hystérique, au même titre qu'une femme, voilà parait-il, qui dépasse l'imagination ${ }^{28}$ ". Associant la sensibilité à la culture, et donc à l'efféminement, Charcot décline le nouveau topos de la dégénérescence fin de siècle : le lien entre la maladie de l'intellectuel et sa manifestation hystériforme. Le regard de démystification athée devient l'outil qui protège le masculin de toute contamination.

\section{ANGOISSES MASCULINES DU FÉMININ}

La division identitaire des genres, étayée par l'école de la Salpêtrière, trouve un écho important dans les théories psychopathologiques au passage du $\mathrm{XX}^{\mathrm{e}}$ siècle. Cette séparation trouve un terreau de diffusion fertile dans les imageries populaires, passant de l'étude médicale à sa condamnation criminelle, relayées par le roman à thèse et son illustration satirique. À la croisée du médical et du culturel, la théorie de la dégénérescence ${ }^{29}$ va creuser le sillon critique de la décadence artistique fin de siècle, dont Péladan est l'une des premières victimes. Dans son fameux pamphlet Dégénérescence, le journaliste Max Nordau s'en prend violemment à Péladan et à

26. Voir Mark S. MiCALE, « Hysteria Male/Hysteria Female : Reflections on Comparative Gender Construction in Nineteenth-Century France and Britain », dans Marina BENJAMIN (dir.), Science and Sensibility. Gender and Scientific Enquiry, 1780-1945, Oxford, Blackwell, 1991, p. 200-239.

27. Jean-Martin ChARCOT et Valentin MAGnAN, « Inversion du sens génital », Archives de neurologie. Revue des maladies nerveuses et mentales, Vol. III, $\mathrm{n}^{\circ} 7,1882, \mathrm{p} .55$ et 56.

28. Jean-Martin CHARCOT, «Lecon 1 » (1885), Leçons sur l'hystérie virile, ouvr. cité, p. 37.

29. Sur ce point, voir notamment Jean-Christophe CofFIn, La Transmission de la folie, 1850-1914, Paris, L'Harmattan, 2003 et Christophe PrOCHASSON, Paris 1900 : Essai d'histoire culturelle, Paris, Calmann-Lévy, 1999. 
ses salons, le rapprochant de Wagner et en le représentant en "dégénéré supérieur " mystique et "émotif sexuel " : "Dans la vie et la conduite des hommes qui écrivent des œuvres mystiques, symboliques et décadentes, dans l'attitude prise par leurs admirateurs, dans les goûts et instincts esthétiques de la société à la mode, on observe la confluence de deux conditions de maladies bien définies, familières au docteur, la dégénérescence et l'hystérie, dont la phase bénigne est appelée neurasthénie ${ }^{30}$."

Péladan répond aux attaques dans Comment on devient artiste?, en lui opposant une conception sacerdotale de l'artiste : le génie créatif doit se construire sur des principes biologiques et ésotériques. L' " aristie ${ }^{31}$ " devient pour Péladan le moyen néologique de conjuguer l'aristocratie artistique et le sacerdoce religieux, par ce syncrétisme susceptible de rénover le mode de vie masculin, en l'éloignant du féminin. Les femmes artistes sont ainsi exclues des salons rosicruciens, où règnent le célibat et le refus du péché de chair : le mage devient le grand prêtre de l'Idée. Péladan emprunte, pour cela, une rhétorique magico-médicale héritée de son propre frère Adrien Péladan. La concordance spirituelle entre les pôles mâles et femelles expliquent tant l'attirance sexuelle que les attributs physiques de chaque sexe : "L'homme est viril par les génitoires et par la parole, mais passif par le cerveau ; tandis que la femme ouverte à la fécondation physique et animique est, à son tour, fécondatrice dans le spirituel $^{32}$. " La femme féconde les idées de l'homme, devenant sa muse génitrice (et non créatrice) à même de stimuler la création masculine. L'artiste doit donc choisir entre le pôle cérébral et le pôle génital, "si l'on veut atteindre au génie. Perdre le sperme, c'est perdre la vigueur intellectuelle et en ce sens, Alcméon avait raison d'appeler le fluide séminal une goutte de cerveau, stilla cerebri ${ }^{33}$ ".

Cette obsession liée aux pertes séminales et à l'onanisme est détaillée dans son étude doctorale sur le phénomène de la spermatorrhée ${ }^{34}$, qui désigne ces pollutions nocturnes, signes de passivité et d'impuissance dont Adrien Péladan semble être atteint. Joséphin Péladan s'inspire fortement de ces résultats et prône la virginité et le célibat, pour pallier la perte de virilité physique et mentale. La figure de l'androgyne devient pour lui l'ultime idéal de beauté physique : «le comble de l'art des formes résout à une fusion harmonieuse des deux sexes à leur puberté ${ }^{35}$. " Mais la double figure du célibat artistique et de ses figures androgynes attise la curiosité de ceux qui y voient des intentions cachées. L'écrivain néo-chrétien Léon Bloy excelle dans cet exercice, autour des commentaires du cycle de La Décadence latine : "Il nous informe exactement qu'il est du petit nombre des hommes qui ont "la volupté décorative".

30. Max Nordau, Dégénérescence, Paris, Bibliothèque de philosophie contemporaine, Alcan, 1894, tome I, p. 391-399.

31. On se reportera à ce sujet aux analyses de Jean-Pierre GUILLERM, « De l'artiste à l'Ariste : Joseph Péladan contre les décadences », Romantisme, n 67, 1990, p. 59-75 et de Pascal RousSEAU, « L'aristie », dans Traces du Sacré, Catalogue d'exposition, Paris, Centre Pompidou, 2009, p. 88-89.

32. Anonyme, Venus Magique, contenant : les théories secrètes et les pratiques de la science des sexes, Paris, Chamuel, 1897, p. 12 (désigné de manière posthume).

33. Euvre posthume du Dr Adrien PÉLADAN fils, Anatomie homologique; la triple dualité du corps humain et la polarité des organes splanchniques, Paris, J. Baillière et fils, 1886, Préface, p. XX-XXI.

34. Adrien PÉLADAN, Traitement homéopathique de la spermatorrhée, de la prostatorrhée et de l'hypersécrétion des glandes vulvo-vaginales, thèse non publiée, Lyon, 1869.

35. Joséphin PÉLADAn, Salon de 1888, Salle III, Paris, Dentu, 1888, p. 50. 
Mais qu'une spermatorrhée d'idéal, venant à se déclarer, lui interdise la fornication sexuelle, il est tout de même en état de prononcer une parole qui "infécondera la

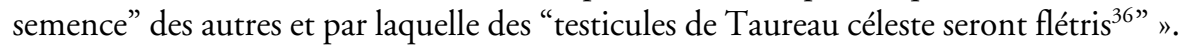
Se moquant de son jeu rhétorique, Bloy souligne aussi l'impuissance supposée au contact d'un trop plein d'idéalisme.

Au célibat combattu au moment où la république réclame la repopulation de sa nation, s'ajoute la lutte farouche contre ceux qui manqueraient aux devoirs familiaux et patriotiques. Dans la caricature de l'illustrateur Mauricio ${ }^{37}$ [fig. 2], les méfaits de cette conception narcissique et ultra-individualiste sont ouvertement exposés : une femme, auprès de son enfant mâle né dans un chou, pleure l'éloignement de l'homme, dont la silhouette rappelle le portrait de Péladan par Marcellin Desboutin, lors du premier salon rosicrucien. Ce rapprochement est confirmé par le halo lumineux, la canne à la main et les nuages entourant le chemin idéaliste. Les larmes qui coulent du visage de l'éplorée ont, pour les besoins de la caricature, une forme proche des gamètes mâles, tombant ainsi sur un sol d'où a émergé le chou. Ce dessin sous-entend à la fois l'abandon et la reproduction issue des larmes versées dans la terre fertile. L'homme, exclu volontaire de la reproduction des nouveaux mâles, ne deviendrait ainsi qu'une image idéelle vers quoi l'avorton pourrait tendre (la direction de sa main en atteste $)^{38}$.

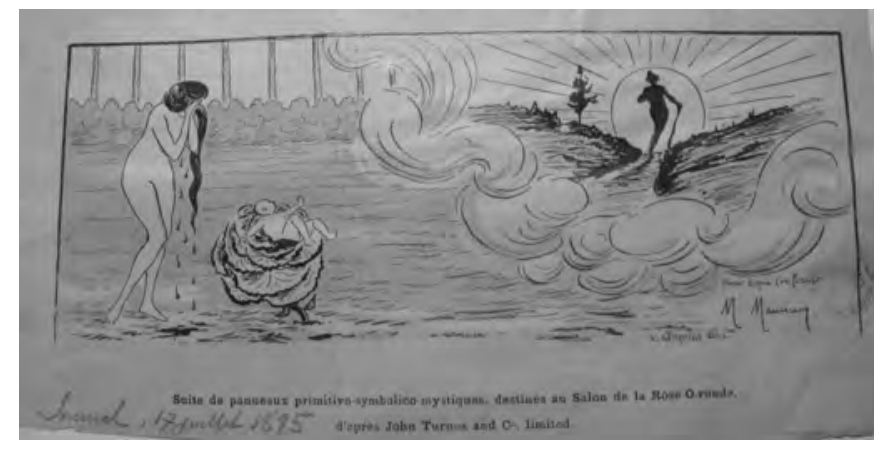

Figure 2. «Suite de panneaux primitivo-symbolico-mystiques », Le Journal, 27 juillet 1895, Fonds Péladan, MS 13412, feuillet.47, BNF.

(D.R.)

L'autre conséquence du retrait idéaliste concerne la diminution physique de l'homme. La caricature du même Mauricio présentant Péladan, tel que l'avait surnommé le Figaro en 1891, "Pèle, Adam ${ }^{39}$ !", se promène vaillamment sur les chemins solitaires. Une femme l'exhorte à « semer », afin de fertiliser sa terre. L'allusion

36. Léon BLOY, « Éloi ou le fils des anges », Gil Blas, 10 juillet 1888.

37. Le Journal, 27 juillet 1895, Fonds Péladan, MS 13412, feuillet 47.

38. Rappelons que la théorie de l'épigénèse a été supplantée à la fin du XIX ${ }^{\mathrm{e}}$ siècle par l'actuelle théorie de l'embryologie.

39. Le Figaro, avril 1891, «Que dit Ève en cueillant la pomme ? Elle dit mon nom : Pèle, Adam ! ». 
à la reproduction sexuelle est soutenue par le geste de la main levée de Péladan et par le port altier, suggérant que la fertilisation n'est pas le ressort d'un effort physique, mais d'une semence idéaliste éjectée de la main. La performance soutenue par les « encore, encore " de la femme se rapproche de celle d'une vie moderne, où la gente féminine soutient le mâle dans la production quotidienne du travail industriel. L'épuisement qui en résulte, ainsi que les demandes ininterrompues, ont un double emploi dans l'imagerie symboliste décriée par le caricaturiste. Le peintre ou écrivain symboliste ne saurait d'une part assouvir les besoins sexuels de la femme, s'épuisant vite à la tâche par sa faiblesse physique et par son esprit débonnaire (le lancé de graine exigeant une tenue affable). L'utilité sociale est d'autre part remise en cause, le symboliste n'étant bon qu'à flâner de manière improductive, sans être capable de travailler, et encore moins de reproduire l'espèce. On retrouve ce même ressort humoristique dans les caricatures des salons rosicruciens, avec Les Âmes déçues de Ferdinand Holder, où les hommes grimés pour l'occasion en hystériques virils de la Salpêtrière, sont totalement amenuisés par les impératifs conjugaux ${ }^{40}$. Le sous-titre souligne les teintes sombres et les mines abattues de la peinture d'origine, dont les raisons sont invoquées à travers les motivations absentes des femmes.

La rhétorique de la dégénérescence, qui dénonce l'intellectuel, s'associe à l'antigermanisme et vilipende "MM. Péladan, Huysmans - il sent son Prussien, ce nom-là - Abel Hermant, Perrin, Octave Mirbeau, Bonnetain, Robert Caze, [qui] ont voulu nous peindre, sous les couleurs les plus odieuses, cette vie d'abnégation, de renoncement et d'hérö̈sme discret, qui est celle des cadres de notre armée ${ }^{41}$ ". Cette crise de l'identité masculine, constituant des figures de rejet par le biais d'une minorité sexuelle, ethnique et politique (la femme, l'homosexuel, l'allemand ou le juif $^{42}$ ) trouve une tournure particulière, dans cette rhétorique de démystification du mystique, à l'instar de la caricature à charge de Vignola ${ }^{43}$ [fig. 3]. Péladan en crucifié accueille des pleureuses nues, sous le regard ébahi des soldats venant du front. La prosternation a des accents de culte sexuel fétichiste, lorsque la femme s'agenouille à ses pieds, pour constater par fanatisme qu'elle touche les chaussures de son maître. L'image de l'écrivain symboliste, crucifié sur l'autel de la décadence, se rapproche de la méfiance envers le cosmopolitisme sémite, dont ces types de caricature prennent le trait. Il s'agit d'un nouvel archétype associant l'antisémitisme aux traits ultra-féminins d'une classe d'intellectuels abandonnant la virilité du combat armé, au profit des combats dans les colonnes des journaux ${ }^{44}$. La féminisation de l'homme est d'autant

40. Fonds Péladan, MS 13412, feuillet 15, non identifié. «Qu'elles ont dû être multicolores les désillusions, sous le poids desquels se sont courbés ces nobles fronts ! Oh ! Les femmes, les femmes ! ».

41. Georges DARIEN et Édouard Dubus, Les vrais sous-offs., Paris, A. Savine, 1900, p. 11.

42. Sur ce processus, voir notamment Georges L. MossE, L'image de l'homme. L'invention de la virilité moderne, Paris, Éditions Abbeville, 1997 et Annelise MAUguE, L'identité masculine en crise au tournant du siècle, Paris, Payot \&Rivages, 2001.

43. Fonds Péladan, MS 13412, feuillet 17, non identifié.

44. Voir en ce sens, l'analyse de Linda Nochlin, « Degas et l'affaire Dreyfus : portrait de l'artiste en antisémite », dans Les politiques de la vision. Art, société et politique au XIXe siècle, Nîmes, Jacqueline Chambon, 1995, p. 201-239. 
plus préoccupante, que sa virilité est en jeu, lorsqu'une littérature ne rend finalement plus hommage à ses vertus morales, mais bien à sa possible chute.

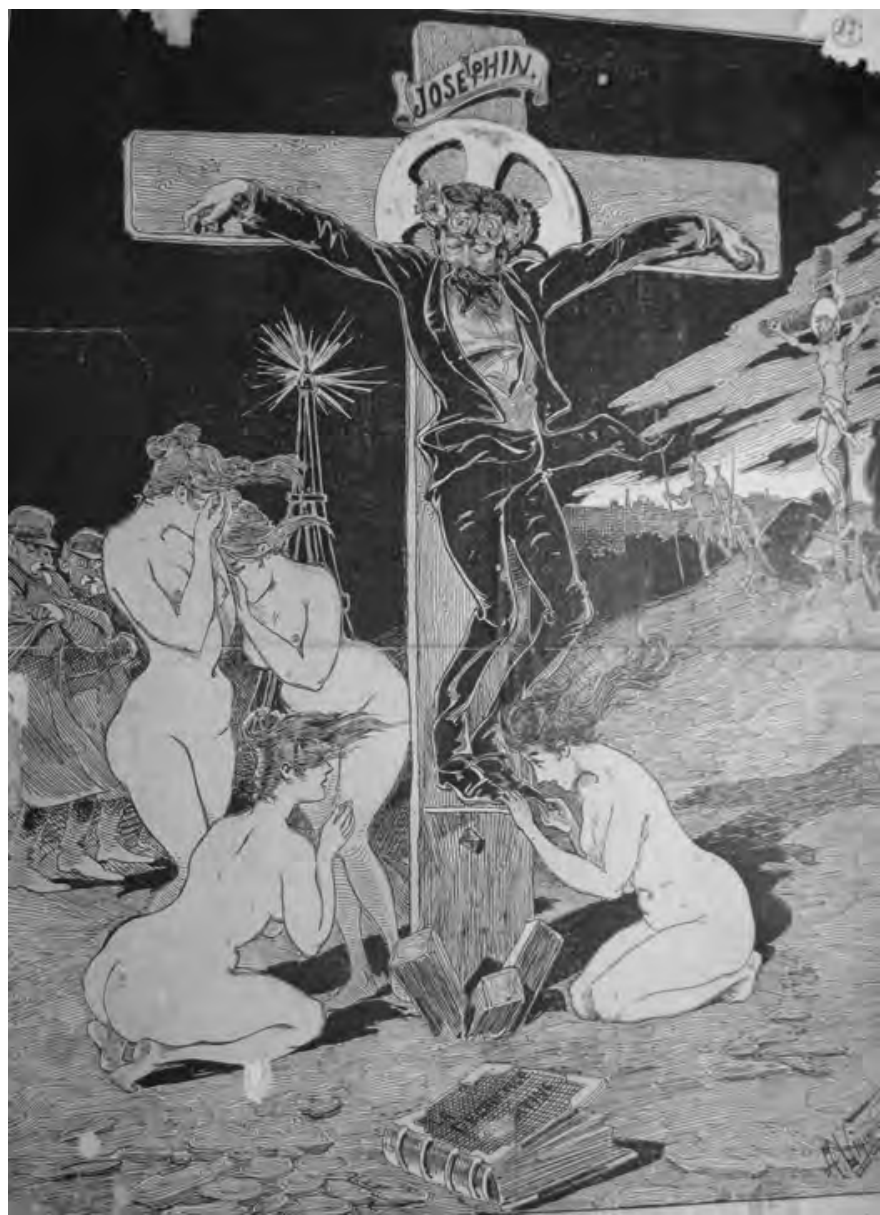

Figure 3. "Josephinus Christus », Fonds Péladan, MS 13412, feuillet 17, BNF.

(D.R.)

\section{« HoMOSEXUALISER » LE MYSTIQUE}

Les vagues néo-chrétiennes traditionalistes vont ainsi se retrouver aux côtés de la critique anticléricale, en associant l'efféminement provoqué par le retrait idéal, aux premiers mouvements de théorisations médicales de l'homosexualitée ${ }^{45}$. L'affaire liée

45. Voir notamment Daniel Borillo et Dominique Collas, L'homosexualité de Platon à Foucault. Anthologie critique, Paris, Plon, 2005 et Sylvie CHAPERON, Les origines de la sexologie. 1850-1900, Paris, Audibert, 2007. 
au journal du Chat Noir développée entre 1885 et 1891 témoigne de ces attaques contre l'ambiguïté débusquée par l'humour montmartrois. Dès l'été 1885, le journal, qui prend l'habitude de nommer un secrétaire de la direction fictif, y fait apparaitre Joséphin Péladan, accompagné du sobriquet " comme la lune ». Si l'expression semble apparaître dans les dictionnaires au début du $\mathrm{XX}^{\mathrm{e}}$ siècle, elle atteste d'un double emploi associant la lune à la distraction, mais aussi, de manière plus lubrique, au postérieur. Le 21 mars 1891, une nouvelle épithète, "derrière éprouvé " pousse Péladan à poursuivre en justice Salis, le rédacteur en chef, pour diffamation et injures publiques. La presse de l'époque suit cette affaire avec délectation, s'interrogeant sur le sens de l'épithète. "De quoi se plaint M. Péladan ? Diable! C'est scabreux à dire. Il y a là-dessous des questions de mœurs dans lesquelles j'aime mieux ne pas m'embarquer ${ }^{46}$. "Salis répond le 18 avril à l'assignation en justice, par une nouvelle expression "derrière récalcitrant ", finissant de rendre l'intéressé hors de lui : "l'injure, cette fois, dépasse tout ", déclare Péladan au journal La France, tandis que son détracteur précise dans ces mêmes colonnes l'objet de ses plaisanteries : "Il y a bien Hugo, Balzac et des tas d'autres, mais pour moi je préfere Péladan... Le mot dont il s'est offensé n'était qu'une façon gauloise chatoiresque d'exprimer cette admiration que je ressens pour lui ${ }^{47}$. " L'affaire se ponctue par un duel auquel Péladan ne peut répondre, redoutant l'excommunication de Rome et le terrassement de son adversaire par ses " pouvoirs magiques ». Le tribunal déboute finalement Péladan, jugeant qu'il n'y avait rien de diffamatoire. Salis finit en l'appelant " Ataxerfesse ", confirmant la série des calembours par cette allusion à son profil assyrien.

L'entreprise idéaliste et ultra-catholique des salons rosicruciens souffre évidemment de cette campagne. Poursuivant son enquête sur le néo-spiritualisme mystique, Jules Bois conclut, non sans espièglerie : « [Péladan] réussit son seul acte public intéressant : l'exposition des Roses-Croix. Encore eut-elle comme résultat non pas tant de révéler les chefs-d'œuvre d'une esthétique nouvelle que de modifier, pendant une année ou deux, la coiffure des femmes. Il s'intitula ensuite le chapelain des vicieuses, et produisit des encycliques où il excommuniait le pape $^{48}$." Rappelant les rapports de Péladan avec les cercles féministes de l'CEillet blanc et ses prises d'armes contre l'Église romaine de Léon XIII, Bois souligne la futilité du projet et la fin programmée du symbolisme décadent. Ce dernier fonde dès 1893 une nouvelle revue Le Cour, avec Antoine de La Rochefoucauld, mécène et peintre ayant rompu avec Péladan dès le premier Salon. Développer un nouvel art religieux passera par les pratiques issues des Nabis et des néo-impressionnistes, et non par ces « anglomanes pasticheurs de préraphaélites ${ }^{49} »$. La disqualification du projet rosicrucien fait se relier l'entreprise décadente littéraire française à l'esthétisme anglais, dont l'affaire du procès Oscar Wilde, en 1895, marque la perte d'influence. Comme le rappelle non sans légèreté

46. Fonds Péladan, MS 13416, feuillet 65, non identifié.

47. Rodolphe SALIS, La France, 27 avril 1891.

48. Jules BoIs, Le Monde Invisible. Les occultistes, les théosophes, le luciférisme, le satanisme, les deux envoûtements, les marchands d'espoir, l'église spirite, les recherches psychiques, conclusions, Paris, Flammarion, 1902, p. 20.

49. TIPHERETH, « L'Art », Le Cour, n 1, avril 1893, p. 6. 
Philippe Jullian, «nos premiers esthètes, Bourget et Barrès, devenaient nationalistes, le premier, pour faire oublier son passé homosexuel et entrer à l'Académie ; le second, après "le culte du moi", n'avait plus rien à attendre de l'esthétisme ${ }^{50}$ ".

La méfiance envers les pratiques homosexuelles n'est pas étrangère à ce que Léo Taxil avait relevé dès $1884^{51}$ : les relations entre hommes se produisent sans distinction de classe sociale et sont censées former une véritable micro-société dans la société civile. Le premier symptôme lié à l'homosexualité est la prostitution et la criminalité ; le second, le désordre moral anti-républicain, également engendré par les groupuscules ésotériques. Cette analyse des pratiques homosexuelles devient un topos dans le réquisitoire contre ce que Proust décrit lui-même comme relevant d'une véritable franc-maçonnerie d'invertis ${ }^{52}$, au moment même où la médecine peine à trouver des moyens pour reconnaître la physionomie d'un homosexuel. Les premiers scandales français vont ainsi associer l'homosexualité au milieu des esthètes. La presse satirique devenue plus agressive, comme la revue L'Assiette au beurre, consacre de nombreux numéros à ce phénomène, démystifiant notamment les activités du comte et écrivain Jacques d'Adelswärd-Fersen qui venait d'être inculpé d'attentat à la pudeur et d'excitation de mineurs à la débauche, en 1903.

L'affaire se retrouve dans le numéro spécial des " Messes noires ${ }^{53}$ ", titre du procès et sobriquet dénonçant les activités ésotérico-sexuelles. L'illustration par Manuel Orazio finit par rassembler toutes les figures de proue du décadentisme fin de siècle avec, au centre, la main levée et couverte de bijoux d'Adelswärd-Fersen, rappelant les excentricités de Jean Lorrain, placé à sa gauche. Jules Bois associe ces événements à des "parodies de messes noires ${ }^{54}$ ", mais ne fait pas de commentaires sur les rapports entre ces jeunes garçons et la présence de nombreuses personnalités parisiennes. Dans le numéro tardif consacré à l'Art Nouveau ${ }^{55}$, la série de dessins présentés par l'illustrateur Raphaël Kirchner fait un parallèle entre les achats de «bibelots " Art Nouveau et les effets physiologiques que cela entraîne. La ligne serpentine des objets et les effets de manipulation proviennent de cet "ars nova suggestiva ", la suggestion hypnotique, où toute personne masculine adoptant ce mode de vie esthète, fondée sur une érotomanie du rapport à l'objet, est vouée à subir des états de mélancolie (associés aux meubles devenus agressifs) et des situations d'apathie sexuelle, n'arrivant plus à faire la distinction entre une femme et un homme [fig. 4]. La série conclut, sans hésiter, que «ces malades ont coutume d'écrire sur l'art moderne, ils deviennent un danger public ». Les mains, bijoutées, en poignets cassés ou évanescentes, deviennent l'apanage des traits physionomiques de l'homosexuel hypnotisant la gente masculine par sa culture d'esthète.

50. Philippe Jullian, Esthètes et magiciens : l'art fin-de-siècle, Paris, Perrin, 1969, p. 241. 1884.

51. Léo TAXIL, La prostitution contemporaine, étude d'une question sociale, Paris, Librairie populaire,

52. Marcel Proust, Sodome et Gomorrhe. À la recherche du temps perdu IV, Paris, Gallimard, 1988, p. $18-19$.

53. « Messes noires », L'Assiette au beurre, $\mathrm{n}^{\circ}$ 141, 12 décembre 1903.

54. Jules BoIs, La Presse, 18 juillet 1903.

55. « Art Nouveau. Jugend-Stil », L'Assiette au beurre, n 339, 28 septembre 1907. 


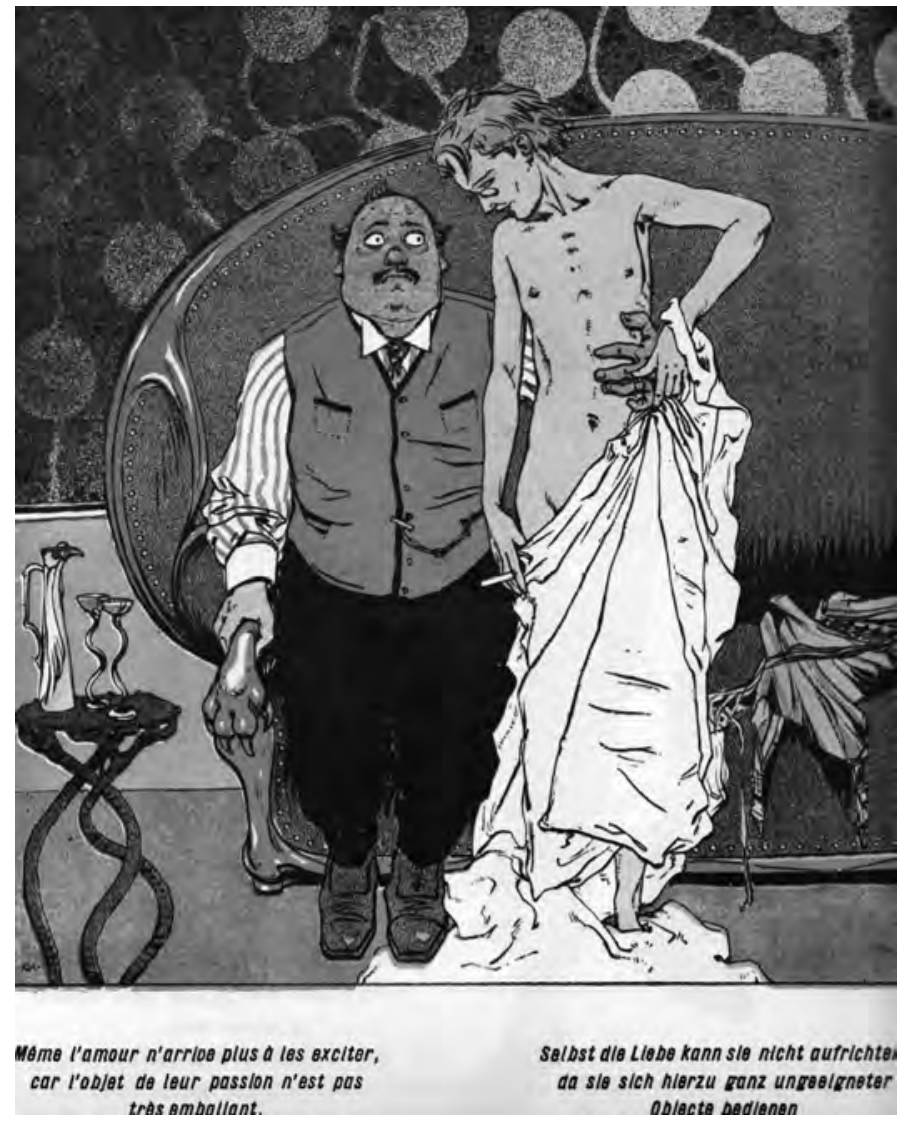

Figure 4. " Art Nouveau. Jugend-Stil », L'Assiette au beurre, n 339, 28 septembre 1907. (D. R.)

Démystifier revient donc à révéler le secret hors du lieu dans lequel il a été conçu, initiant ainsi toute une théorie du secret analysée par Ėve Kosofsky Sedgwick ${ }^{56}$. Péladan s'amuse de ces procédés, commentant dans une lettre à son ami critique William Ritter, ouvertement homosexuel : "On a dit - j’ai le nom, par écrit, quelque part - à Carmen Sylva, que j'étais pédéraste... elle l'a cru, la pauvre femme... [...] mon cher Ritter, je ne serais pas digne de votre admiration si je manquais de cette bravoure à la plume qui écrit humanisme au lieu de patrie, quand telle est la pensée. Croyez à ma sérénité de Mage ${ }^{57}$. " Péladan enfumera donc la vérité sur sa sexualité, qui importe finalement peu, démontrant que l'ambiguïté sexuelle constitue plus

56. Ève Kosofsky SEDGWICK, Épistémologie du placard, Paris, Éd. Amsterdam, 2008 (traduit de l'anglais par Maxime CERVulle, Epistemology of the closet, Berkeley, University of California Press, 1990).

57. Lettre de Joséphin Péladan à William Ritter du 30 décembre 1889, ALS, f. WR, lot 19, cité dans Xavier Galmche, «William et les garçons (d'Europe centrale) "Aventures de volupté sous d'autres cieux" », en ligne sur le site du CIRCE, consulté le $1^{\text {er }}$ octobre 2011. 
un ressort polémique qu'une vérité à réclamer. S'y agitent les soubresauts d'une époque fulminant la décadence, à travers les bouleversements d'une société éliminant tout groupuscule à l'écart de l'unité républicaine. L'idéalisme alternatif de Péladan annonce en filigrane la régénérescence d'une masculinité début de siècle, qui se jouera de la moralité ordonnée par l'anticléricalisme.

(Université Paris 1 Panthéon-Sorbonne) 